\title{
Analysis of Different Routing Techniques for Opportunistic Data Transfer
}

\author{
Sheela Rani Arasu \\ Post Graduate Scholar \\ Department of Computer Science and Engineering \\ Karunya University, India
}

\author{
Immanuel Johnraja Jebadurai \\ Assistant Professor (SG) \\ Department of Computer Science and Engineering \\ Karunya University, India
}

\begin{abstract}
This paper deals with various routing techniques for efficient data transfer in MANET. MANET is a wireless infrastructureless network where mobile devices communicate through wireless links. To make the communication of wireless network as good as that of the wired ones is the challenging issue. The high link quality involves best path selection and it leads to throughput improvement. Higher layer like network layer is used to provide an efficient data transfer in dynamic environment. To make routing efficient, we need to know the topographical information of the wireless network. Based on which we can improve the routing process and packets are transmitted efficiently with high packet delivery ratio. This paper shows an analysis of various techniques for improving the link quality with best path selection for opportunistic data transmission with its advantages and disadvantages.
\end{abstract}

\section{General Terms}

MANET, Routing.

\section{Keywords}

AODV, DSDV, DSR, ExOR, GPS, Network Layer, OLSR, Packet Delivery Ratio, PSR, QOS.

\section{INTRODUCTION}

Mobile ad hoc network is a dynamic environment where communication between mobile devices takes place through wireless links. There is no central controller authority which takes care of transmission between two nodes or in other words, it is an infrastructure less network. In MANET each node is capable of acting both as routers and hosts [12]. Since it is not a static environment each node is independent and free to move in any direction which results in change of links to other devices more often. In MANET when a node is not in direct communication, it takes help of other nodes to forward the data. Transmission is through multihop wireless links. Since the network is decentralized both the work of finding topology information and deliverance of data to the destination is done by the nodes itself. MANET is a standalone network and is quickly deployable. It is a self organizing and reconciling network.

Each node is responsible for the management of the network. In dynamic network, Topology changes occur swiftly and unpredictably. Network topology tells about the arrangement of nodes including its connecting units. In static environment there is no movement of nodes. Topology control [1] is needed to determine appropriate topology in ad hoc network which saves energy, reduce interference between nodes and extends the lifetime of the network. Based on connectivity, energy efficiency, throughput, robustness to mobility, the quality of topology is determined. High level routing protocols are implemented over a suitable topology.

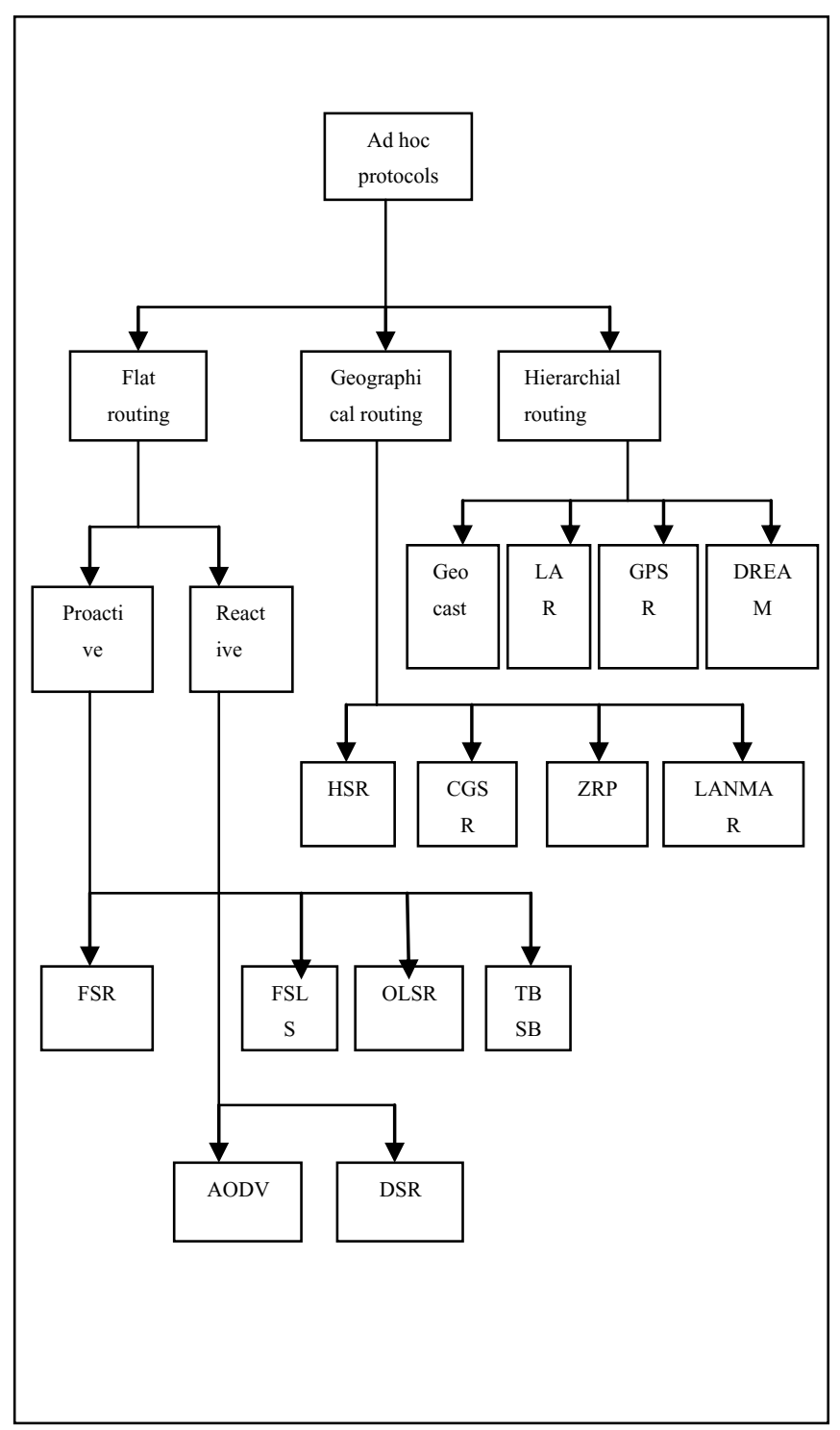

Fig 1: Routing Protocols Classification

All connected nodes must act as routers to support in accurate delivery of data packets. As routers, node must contribute in detection and maintenance of routes in network. Node must endlessly work to find new nodes, broadcast new node and route information to other linked nodes, identify and report on nodes that are no longer connected to the network. Routes contain links which is the connection between two nodes. A 
change in link quality influences the route quality. A route consisting of varying link does not produce good results.

There are various routing protocols available for data transfer. Flat routing protocol [13] is used to find out the finest route hop by hop to a destination by means of any path. Hierarchical routing protocol forms the network as the hierarchy of nested cluster of nodes. In geographical routing protocol, instead of network address, a position is used for sending data from source to destination. Figure 1 shows the classification of different routing protocols [9]. On-Demand protocols (Reactive protocol) like AODV and DSR is based on hop counts. Table driven protocols like (Proactive protocols) like DSDV, OLSR are based on link states.

\section{ROUTING TECHNIQUES FOR OPPORTUNISTIC DATA TRANSFER}

This section tells about various routing techniques for opportunistic data transfer with its merits and demerits.

\subsection{Location based Protocols}

In position based opportunistic routing [3], the location information of the node can be acquired by geographic position system (GPS) and the neighbours are updated at regular intervals. Global Position System [14] provides data about location and time in all weather anywhere on or near the earth. When a source has a packet to send to a destination, first a forwarder list is calculated based on the distance between its neighbours and destination. The packet header contains this forwarder list. Higher priority is given to the node near the destination. Next hop is chosen based on the best forwarder. The information is sent to all nodes that is one hop distance from the source. The forwarder list is checked by the receiver. If there are $\mathrm{n}$ slots before it then it will wait for $\mathrm{n}$ time slots before it forwards the packet. A node discards the packet if it hears the same packet being sent by other node. Neighbour list, ID record, forwarding table, packet list and packet buffer information are maintained. Link failures and attacks are avoided. It is more robust and efficient. The disadvantage is that it requires more amount of buffer space.

Vehicular network [7] is an example of hybrid delay tolerant network which uses infostations and vehicles to geographically route information to the destination. It uses a navigation system that consists of global positioning system to route a data packet to the correct location. Vehicles are mobile sensors that gather information about the traffic and road conditions. This information is sent to the local infostation. Local infostation gathers information from many sources and estimates the traffic condition and this information is sent to the other vehicles. The vehicle that receives this warning information calculates an alternate route to avoid the congested route. In case of remote areas there is no connectivity between the vehicles and infostation and other vehicle act as a data mule to forward this information to the infostation. When a vehicle follows a suggested route it finds a nearest point to reach the destination of a packet. Using this nearest point and global positioning system it calculates a utility function that estimates the minimum estimated time to reach the destination. A vehicle that can forward the packet quickly to the destination becomes the next carrier of the packet. The minimum estimated time for delivery to reach the destination (METD) is equal to the sum of estimated time to reach the nearest point and estimated time from the nearest point to destination. There is a god performance in terms of delivery ratio, delay and overhead. This technique can be compared with location based greedy routing and $\mathrm{MoVe}$ routing algorithm. According to greedy algorithm [19][20] the packet is forwarded to the neighbour that is closest to the destination. This process is repeated until the message reaches its destination. MoVe algorithm [18] uses information about relative velocities of the current vehicle and the neighbours to predict the closest distance that the vehicles are predicted to get to the destination following their current trajectories. Optimization, privacy and security aspects are not considered in this method.

\subsection{ExOR: Opportunistic MultiHop Routing for Wireless Networks}

ExOR [2] is an integrated routing and MAC technique that realizes some of the advantages of cooperative diversity on standard hardware such as 802.11. ExOR broadcasts the packet and decides who forwards the packet only after reception.

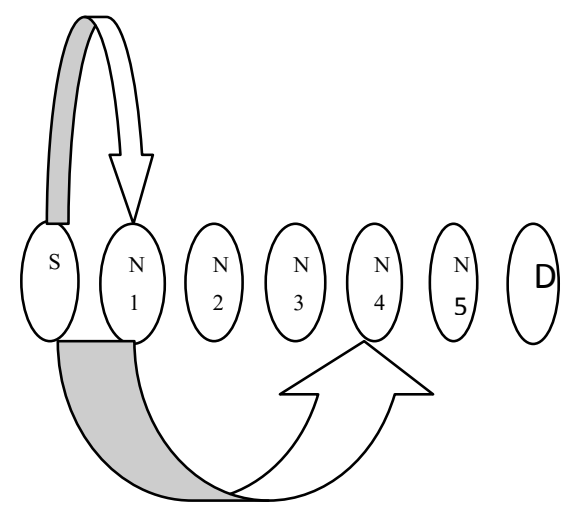

Fig 2: Throughput improvement

ExOR is more beneficial compared to traditional routing. It improves the throughput. The source node broadcasts a packet and some subset of the nodes gets this packet. The nodes run a protocol to find and decide which nodes are in the subset. Those subset nodes that are near to destination broadcast the packets. ExOR has a metric reflecting the cost of transferring packet from any node to destination. This cost metric is same as ETX [15] but the difference is ExOR uses only the forward delivery probability. This manner continues until the packet is received by the destination. ExOR avoids interference or duplication. The ExOR header follows the Ethernet header followed by packet's data which includes the batch size, forwarder list size, packet number, forwarder number, batch map and fragment. ExOR avoids multiple nodes [21] transmitting the same packet to the destination node by tying the MAC to the routing, commanding a strict scheduler on routers access to the medium. Even though the medium access scheduler provides suitable throughput gains, it happens so at the cost of losing some of the suitable features of the current 802.11 MAC. Moreover, this greatly organized approach to medium access makes the protocol difficult to extend alternate traffic types mainly multicast. There is also a spatial reuse lost [24]. MORE in contrast to ExOR randomly mixes the packet before forwarding it and it uses network encoding which restricts the amount of retransmission and recovery overhead 
[23]. Network encoding allows [22] a node to send out packets that are linear combination of the previously received information. MORE performance is good but not much better than ExOR under more realistic scenarios.

Figure 2 shows how ExOR uses the transmission which is failed in traditional routing. The transmission path is from $\mathrm{S}$ to $\mathrm{N} 2$ to $\mathrm{D}$. If the transmission falls before N2, ExOR can use that whereas in traditional routing this transmission is missed out and source must retransmit. If the transmission falls after $\mathrm{N} 2$ then traditional routing cannot use that luck also but ExOR make use of this transmission. The disadvantage of this technique is that it is not scalable to large network and there is an overhead in packet header.

\subsection{PSR: Proactive Source Routing in Mobile Ad Hoc Network}

Proactive Source Routing Protocol [4] is much well organized when compared with distance vector based protocols. Each node contains the entire structure information about the network than distance vector based protocols. It does not contain timestamp links. In PSR each node has a breath first spanning tree (BFST) which will be responsible for providing data transportation services. For opportunistic routing in MANET, source routing concept is used and thus intermediate nodes just forward the packet and need not look at its own routing table for information [10] and which is the main advantage of source routing. Nodes regularly broadcast the network structure information to the finest of its awareness. This helps a node to expand the capacity of the knowledge about the network which results in best path selection. PSR maintains a BFST at each node and some challenges has to be taken care of and they are overhead reduction, high data transportation performance and loop prevention. Some more issues that have to be noted are incorporation of neighbour trees and notification of unreachable nodes. It has small communication overhead. In case of error, the intermediate which has the packet drops the packet which has to be avoided by choosing some quick local repair policy which is also another disadvantage.

PSR is highlighted by proactive source routing, small routing overhead and loop free. The efficiency of opportunistic data forwarding [5] depends on the proper routing protocol. PSR is an efficient routing protocol. In PSR each node broadcast the tree structure and thus the nodes update the network topology information. This update information is iterative and scattered among all nodes. The regular broadcast of routing messages in PSR also doubles as Hello messages for a node to discover which other nodes are within its range. The neighbour trimming procedure is provoked at each neighbour when there is no routing update or no data packet has been received from this neighbour for some time, or when a data transmission to node has failed as reported by the link layer. Full update messages are sent less frequently than shorter messages containing the difference between the current and previous states of a node's routing module. The disadvantage is that in case of any error the node drops the packet. There is no information on the retransmission of the dropped packet in case of any error.

\subsection{On-Demand Link Weight Protocol}

This protocol [8] is based on QOS parameters such as bandwidth, delay and node lifetime. When a source node has a data to send to destination, it first broadcasts the route request (RREQM) message to all its neighbouring nodes. The nodes that receives this RREQM message compares this message with its other entire RREQM message received from neighbouring nodes. A forwarding node forwards this RREQM only when it meets the QOS requirements. The destination node after receiving all the RREQM messages which contains QOS information from neighbouring sender, returns a route reply (RREPM) message only when its route meets the needed bandwidth, low accumulated delay and long route lifetime. Thus a reliable route can be provided but route calculation takes little delay. If two or more routes have a same bandwidth then a route with low accumulated and longer route lifetime will be chosen for data transfer [16][17].

\subsection{Energy Consumption Routing for Mobile Ad Hoc Network}

Energy Consumption Routing [11] is loop free adaptive path which tries to reduce both routing and storage overhead. This is responsible for efficient robustness to host mobility, optimization of network resources and flexibility to wireless channel fluctuations. When a source node wants to communicate to destination node it checks it route cache for a route to transfer data to destination. In case of no path available to destination it invokes a route discovery mechanism which is broadcasting a request packet to its neighbours. Based on four metric such as nodes battery power, nodes stability, link quality and availability of the link using future prediction for the link's state, a node is selected for data transfer. Thus route is accumulated in the packet header until it reaches the destination node. The destination on receiving this request, send a reply to the source node based on the accumulated route in the header in reverse direction. The source node stores this route in cache. It avoids channel overhead and improves scalability.

\subsection{Link State Routing}

Link vector algorithm [6] is used for the maintenance of routing information. Each node maintains a topology that it knows the next best hop to reach the destination. Based on this subset of topology information the node can find the best path for communication. Update is performed. This method is used when a correct and deterministic algorithm to select paths are known. The idea here is that each router reports to its neighbours the characteristics of each of the links it uses to reach destination through one or more preferred paths. It also reports to its neighbours about which link it has erased from its preferred paths. Using this information each router construct a source graph consisting of all links it uses in the preferred paths to the destination. It is necessary and it must be shown that each neighbour contains information about the updates about the link and it is consistent.

\section{CONCLUSION}

An analysis on various methods for opportunistic routing is done and comparison is shown. Each method has its own advantages and disadvantages. PSR could be considered as good among the others but in case of packet drop some technique has to be used for retransmission with little delay. ExOR has high throughput and good delivery ratio. 
Table 1. Comparison of various routing techniques

\begin{tabular}{|c|c|c|c|c|}
\hline $\begin{array}{c}\text { Routing } \\
\text { Protocols }\end{array}$ & Delay & $\begin{array}{c}\text { Packet } \\
\text { Delivery Ratio }\end{array}$ & Overhead & Throughput \\
\hline $\begin{array}{c}\text { Location } \\
\text { based } \\
\text { Protocols }\end{array}$ & Low & Good & High & High \\
\hline Tables & Low & Good & Medium & High \\
\hline Tables & Low & Medium & Small & - \\
\hline Tables & Medium & - & - & - \\
\hline Tables & Low & - & Less & - \\
\hline Figures & Average & - & Low & - \\
\hline
\end{tabular}

\section{REFERENCES}

[1] Rajaraman, R. "Topology Control and Routing in Ad hoc Networks: A Survey," SIGA News, vol. 33, pp. 60-73, June 2002.

[2] Ding, W., Biswas, S., and Morris, R., "ExOR: Opportunistic Multi-Hop Routing for Wireless Networks," in Proc. ACM Conference of the special Interest Group on Data Communication, Philadelphia, PA, USA, August 2005, pp. 133-144.

[3] S. Yang, F. Zhong, C. K. Yeo, B. S. Lee, and J. Boleng, "Position Based Opportunistic Routing for Robust Data Delivery in MANETs," in Proc.2009 IEEE Conference on Global Telecommunications (GLOBECOM), Honolulu, Hawaii, USA, December 2009, pp. 13251330.

[4] Z. Wang, C. Li, and Y. Chen, "PSR: Proactive Source Routing in Mobile Ad HocNetworks," in Proc. 2011 IEEE Conference on Global Telecommunications (GLOBECOM), Houston, TX USA, December 2011.

[5] Z. Wang, Y. Chen, and C. Li, "A New Loop-Free Proactive Source Routing Scheme for Opportunistic Data Forwarding in Wireless Networks," IEEE Communications Letters, to appear.

[6] J. Behrens and J. J. Garcia-Luna-Aceves, "Distributed, Scalable Routing based on Link-State Vectors," in Proc. ACM SIGCOMM, 1994, pp. 136- 147.

[7] Leontiadis and C. Mascolo, "GeOpps: Geographical Opportunistic Routing for Vehicular Networks," in Proc. IEEE International Symposiumon a World of Wireless Mobile and Multimedia Networks (WoWMoM), Helsinki, Finland, June 2007.

[8] S. Murthy and J. J. Garcia-Luna-Aceves, "An Efficient Routing Protocol for Wireless Networks," Mobile Networks and Applications, vol. 1, no. 2, pp. 183-197, October 1996.

[9] Tanu Preet Singh, Shivani Dua and Vikrant Das, "Energy Efficient Routing Protocols in Mobile Adhoc Networks", vol 2, issue 1, January 2012.

[10] Kestina Rai, Rubinder Kaur and Kanchan Aggarwal, "Operation of Dynamic Source Routing in Wireless Mobile Ad Hoc Networks".

[11] Hasnaa Moustafa and Houda Labiod, "Energy Consumption Routing for Mobile Adhoc Networks".
[12] Anne Aaron, JieWeng, Performance Comparison of Adhoc Routing Protocols for Networks with Node Energy Constraints International Journal of engineering and technology, EE 360 Class Project Spring 2000-2001.

[13] [online]http://www.inetdaemon.com/tutorials/internet/ip/ routing/flat_vs_hierarchical.shtml

[14] [http://en.wikipedia.org/wiki/Global_Positioning_System

[15] D. De Couto, D. Aguayo, J. Bicket, and R. Morris. "A high-throughput path metric for multi-hop wireless routing", In Proc. ACM/IEEE MobiCom, September 2003.

[16] A N Al-Khwildi, \& H S Al-Raweshidy "A Proficient Path Selection (Link-Weight) Algorithm for a Novel OnDemand Link-State Wireless Ad Hoc Routing Protocol”, WWRF15, Paris 2005.

[17] N Al-Khwildi, \& H S Al-Raweshidy" A Proficient Path Selection Wireless Ad Hoc Routing Protocol", IEEE ICAT 8th International, Phoenix Park. Korea 2006.

[18] J. Lebrun, C.-N. Chuah, D. Ghosal, and M. Zhang. "Knowledge-Based Opportunistic Forwarding in Vehicular Wireless Ad Hoc Networks", Vehicular Technology Conference, 2005. VTC 2005-Spring. 2005 IEEE 61st, 4:2289-2293, 2005.

[19] C. Lochert, H. Hartenstein, J. Tian, H. Fussler, D. Hermann and M. Mauve, "A routing strategy for vehicular ad hoc networks in city environments", Intelligent Vehicles Symposium 2003. Proceedings. IEEE, pages 156-161, 2003.

[20] M. Mauve, J. Widmer, and H. Hartenstein. "A survey on position-based routing in mobile ad hoc networks", IEEE Network, 15(6):30-39, 2001.

[21] http://people.csail.mit.edu/dina/pub/MORE.pdf

[22] C. Fragouli, J.-Y. L. Boudec, and J. Widmer, "Network Coding: an Instant Primer," SIGCOMM Computer Communication Review, vol. 36, pp. 63-68, January 2006.

[23] Daniel Strommen, Trading Structure for Randomness in wireless Opportunistic Routing. http://pages.cs.wisc.edu/ akella/CS740/S08/S08reviews/ CJK+06.pdf.

[24] Szymon Chachulski, Micheal Jennings, Sachin Katti and Dina Katabi, "Trading Structure for Randomness in Wireless Opportunistic Routing”, August 18, 2011. 